\title{
Impacto das parametrizações de microfísica e de camada limite planetária na simulação de eventos meteorológicos intensos em Santa Catarina usando o modelo WRF
}

\author{
Gerson Luiz Camillo ${ }^{1}$ and Dirceu Luis Herdies ${ }^{2}$
}

\begin{abstract}
Resumo
Eventos extremos associados a fenômenos meteorológicos são ocorrências que causam estragos materiais e impactos socioeconômicos diversos. Esses eventos têm ciclo de vida curto, em geral, até duas horas, mas que podem se estender por até seis e 12 horas. Existem diversas abordagens para previsibilidade e uma delas faz uso de modelos numéricos de previsão de tempo. Neste trabalho se quer investigar o conjunto de parametrizações de camada limite e de microfísica que melhor respondem à previsão de eventos atmosféricos intensos. Será usado o modelo de código livre Weather and Research Forecasting (WRF) para fazer os experimentos e a metodologia será por meio de estudo de caso de evento extremo ocorrido no oeste catarinense em 14 de agosto de 2020. Ao final, espera-se encontrar uma configuração do WRF que possa ser usada operacionalmente para predizer e monitorar eventos atmosféricos intensos.
\end{abstract}

\section{Palavras-chave}

Eventos, Extremos, WRF

\section{Introdução}

Eventos atmosféricos intensos são manifestações da atmosfera na forma de precipitação intensa, granizo, ventos fortes associados ou não a rajadas e tornados. Estão associados a nuvens cumulonimbus e estas podem se formar de forma isolada ou em conjunto. Elas podem se organizar de diversas formas, e uma delas é em forma de linha de instabilidade. As linhas de instabilidade pré-frontais são um conjunto de nuvens, especialmente cumulonimbus, em forma de linha, que se desenvolvem e avançam na dianteira de um sistema frontal (Reboita, 2012). As linhas de instabilidade também podem se formar em conjunto aos sistemas convectivos de mesoscala (SCM), representando igual nível de atividade de tempo severa. Com o tempo foram sendo desenvolvidos diversas técnicas para prever a intensidade, deslocamento e área de atuação desses sistemas. Um dos mais usados é a modelagem numérica de tempo.

Modelos numéricos de previsão de tempo (em inglês, Numerical Weather Prediction - NWP) constituem a ferramenta primordial para a previsão do estado futuro da atmosfera. E, a partir das principais variáveis atmosféricas prognosticadas, estimar os principais fenômenos atmosféricos, a precipitação, ventos, nebulosidade, etc. O processo de modelagem parte do instante no tempo no qual o estado da atmosfera deve ser o mais preciso possível. Esse instante corresponde ao conjunto de observações atmosféricas globais, do ar à superfície, do ar superior e da superfície do mar.

As formulações físico-matemáticas permitem discretizar as equações que regem o comportamento da atmosfera e seus processos em altas resoluções espaciais (horizontais e verticais), entretanto, isso demanda o uso de supercomputadores, com alto rendimento computacional, o que limita o uso de altas resoluções espaciais. Entram em cena as parametrizações, conjunto de formulações matemáticas ideais para determinados processos. A questão é que para cada tipo de processo físico a modelar, há um conjunto de parametrizações à escolha. Além das parametrizações interagirem entre si, questões geográficas (latitude, orografia) e de modelo (tamanho de grade, quantidade de níveis na vertical) impactam nas previsões dos eventos atmosféricos.

Um modelo que pudesse fornecer entre 6 e 12 horas antes uma informação sobre condição para tempo severo poderia ajudar na decisão dos órgãos de monitoramento e de defesa civil. Para isto é necessária uma configuração de modelo que tenha a melhor sensibilidade para prever o desenvolvimento destes tipos de sistemas convectivos.

Desta forma, quer-se investigar a sensibilidade do modelo Weather and Research Forecasting (WRF) (Skamarock, 2019) para diferentes parametrizações de camada limite planetária (PBL) e de microfísica de nuvens (MP) considerando os efeitos sobre índices de estabilidade e

${ }^{1}$ Instituto Nacional de Pesquisas Espaciais (INPE)

${ }^{2}$ Progrmaa de Pós Graduação em Clima e Ambiente, Instituto Federal de Santa Catarina, Florianóplois

Email: dirceu.herdies@inpe.br (Dirceu Luis Herdies)

\section{Corresponding author:}

Gerson Luiz Camillo, Instituto Nacional de Pesquisas Espaciais (INPE) Email: gerson.camillo@gmail.com 
variáveis atmosféricas indicadoras de probabilidade de convecção profunda e violenta.

A proposta do presente trabalho visa indicar configurações do modelo WRF que podem resultar em simulações mais realistas de eventos severos considerando a área geográfica do Estado de Santa Catarina. Com isso, haveria disponibilidade de mais uma ferramenta que pode ajudar a melhorar os sistemas de alerta de tempo severo e, em consequência, minorar perdas materiais e de vidas.

\section{Revisão Bibliográfica}

A atmosfera terrestre possui um estado e este pode ser quantificado através de um conjunto de variáveis, dentre elas a temperatura, a umidade, a pressão e o vento. As mudanças no estado atual para um estado futuro são governadas por equações diferenciais parciais da dinâmica de conservação da massa (hidrodinâmica em particular), termodinâmica e dos gases (Bauer, Thorpe, \& Brunet, 2015). Mas muitos processos atmosféricos não podem ser diretamente modelados e representados por variáveis e equações considerando a falta de resolução e na complexidade desses processos, o que demandaria muito tempo computacional para considerar todas as interações (Stensrud, 2007; Warner, 2011).

As equações devem ser resolvidas numericamente e as discretizações espaciais e temporais não permitem obter soluções analíticas para todos processos e interações na atmosfera. Os processos que não podem ser resolvidos explicitamente são tratados através de esquemas de parametrização (Bauer et al., 2015). São simplificações da física e da matemática desses processos que permitem a execução e a obtenção de resultados em tempo útil. Atualmente, as parametrizações que são relevantes à previsão de tempo estão ilustradas na Figura 1, conforme Bauer, Thorpe e Brunet (2015, p. 48). Há alguns fatores relacionados às parametrizações e que são importantes na acuracidade das previsões. O primeiro aspecto é que várias interagem entre si diretamente e algumas delas possuem diferentes formulações para diferentes escalas espaciais. Portanto, estudos de sensibilidade procuram determinar conjuntos de parametrizações que melhor respondem em uma área geográfica para determinados fenômenos. Há casos em que estações do ano também podem impactar no nível de predição.

O WRF possui um conjunto grande de parametrizações, geralmente várias para cada processo físico a modelar. Duas dessas parametrizações vêm sendo investigadas na literatura (Comin, Justino, Pezzi, de Sousa Gurjão, Shumacher, Fernández \& Sutil, 2021; ) (Efstathiou, Zoumakis, Melas, Lolis, \& Kassomenos, 2013; Moya-Álvarez et al., 2020; Schwitalla, Branch, \& Wulfmeyer, 2020) como importantes na acurácia da previsão de eventos de chuva intensos. A primeira e mais importante é a parametrização de microfísica de nuvens (MP) e ela inclui a resolução explícita do vapor de água, de nuvens e dos processos de precipitação (todos os tipos) (Skamarock, 2019; Warner, 2011). Sua escolha determina não somente a quantidade como também o padrão de distribuição espacial de precipitação, principalmente nos modelos regionais (Chawla, Osuri, Mujumdar \& Niyogi; 2018).
A camada limite planetária é a porção inferior da atmosfera de altura de poucos metros a alguns quilômetros na qual os processos de fluxos de energia são mais intensos. É a parte da troposfera que é diretamente influenciada pelo regime de aquecimento solar e resfriamento, estando intimamente ligada à superfície terrestre. Pela complexidade dos processos que nela ocorrem, ela também é parametrizada nos modelos numéricos (Stensrud, 2007). A parametrização de camada limite planetária (PBL) exerce papel fundamental na previsão de precipitação (Shin \& Hong, 2011). Alguns autores investigaram a sensibilidade de modelos na previsão de precipitação considerando somente os diferentes esquemas de PBL (Moya-Álvarez et al., 2020).

As parametrizações de MP e de PBL foram avaliadas no WRF para simular eventos intensos de precipitação em

localidade ao norte da Grécia (Efstathiou et al., 2013).

Foram testados dois esquemas de PBL (MYJ e YSU) e três esquemas de microfísica (Purdue Lin, WSM6 e ETA), totalizando seis experimentos. Foram definidos três domínios aninhados, tendo o externo cobrindo boa parte da Europa e norte da África $(27 \mathrm{~km})$, enquanto os outros dois domínios cobriam áreas da Grécia $(9 \mathrm{~km})$ e a de maior resolução (3 $\mathrm{km}$ ), o norte do país. Os diferentes esquemas de PBL e MP tiveram diferenças quanto à distribuição e intensidade da precipitação, mas as precipitações mais intensas foram melhor simuladas pela parametrização MP ETA e PBL YSU.

Os autores C Chawla, Osuri, Mujumdar, and Niyogi (2018) investigaram o WRF quanto à simulação de eventos de chuva extrema na bacia do rio Ganga. Foram feitos experimentos com quatro tipos de parametrizações de microfísica, duas de cumulus, duas de PBL e duas opções de superfície, além de diferentes resoluções de grade.

Os resultados apontaram para o seguinte conjunto de parametrizações: MYJ (PBL), BMJ (cumulus) e Goddard (MP). Outros achados: identificaram a influência do parâmetro de (PBL) na modulação da magnitude da precipitação enquanto o padrão de distribuição dela sendo impactada pelo fator MP. Um outro aspecto relevante foram os melhores resultados para o conjunto de duas grades de 27 e $9 \mathrm{~km}$, quando comparado com os domínios de 27 e $3 \mathrm{~km}$. Apesar deste último trabalhar com grade de resolução maior, os processos de interação (downscaling) possuem melhores respostas nas proporções de grade menores, como foi o caso do primeiro conjunto, de 3 para $1(27: 9 \mathrm{~km})$.

A convecção sobre a península Arábica foi estudada por Schwitalla, Branch e Wulfmeyer (2020) através do modelo WRF. As parametrizações testadas foram a PBL e a MP e o objetivo era verificar qual conjunto reporta melhores resultados de potencial de desenvolvimento convectivo intenso. A consequência é a possibilidade de eventos intensos de precipitação e ventos fortes. Os melhores resultados foram obtidos pelos esquemas MYNN (PBL) e Thompson (MP).

Tendo como região de interesse a região geográfica das montanhas do Peru, Moya-Álvarez, Estevan, Kumar, Rojas, Ticse, Martínez-Castro e Silva (2020) fizeram experimentos com o modelo WRF mas considerando apenas a parametrização de camada limite planetária (PBL). Foram definidas duas grades com resolução espacial de 18 e $6 \mathrm{~km}$. A orografia acidentada da área é uma questão sensível para os modelos numéricos e, em última análise, na definiçãodos 


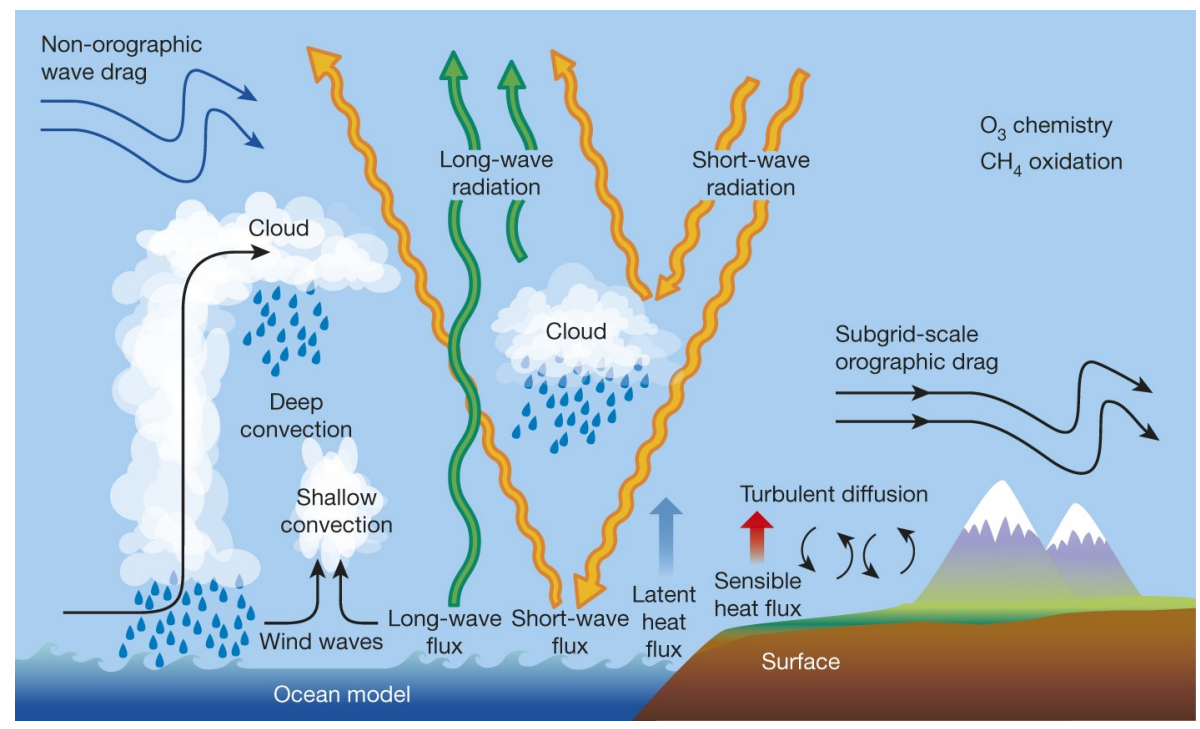

Figure 1. Processos físicos importantes para a previsão de tempo e que são resolvidos por parametrizações (Bauer et al., 2015).

diversos parâmetros. A intensa variabilidade das elevações pode refletir na energia das ondas na solução das equações do modelo, o que pode gerar o desenvolvimento de instabilidades não-lineares (Warner, 2011). Nas definições dos limites das grades, devem ser evitados que atravessem áreas de relevo acidentado, pois podem gerar resultados errôneos.

A pesquisa tratou de averiguar um total de dez esquemas de PBL. De forma geral, todos as parametrizações levaram a uma estimativa abaixo da quantidade de precipitação sobre os Andes Peruanos, em média sete por cento. O esquema MYJ (Mellor-Yamada-Janjic) foi o que apresentou a média de precipitação mais próxima à observada, mas também foi o que apresentou a menor variabilidade (logo, menos sensível a extremos de precipitação). $\mathrm{O}$ esquema $\mathrm{BL}$ ou BouLac (Bougeault-Lacarrère) foi o que apresentou maior variabilidade, mas neste caso foi além do que foi registrado pelas observações de precipitação.

Um dos trabalhos mais recentes no sentido de estudar tipos de parametrizações e seu impacto na estimativa de precipitação, foi dos autores Comin, Justino, Pezzi, de Sousa Gurjão, Shumacher, Fernández \& Sutil (2021). Usaram o modelo WRF para estimar previsões de eventos de chuva extremos no Nordeste do Brasil, mais especificamente na região litorânea. Foram definidos como fatores a investigar o conjunto de parametrizações de microfísica (MP) e de camada limite planetária (PBL). Foram seis esquemas de MP e três de PBL considerando três grades aninhadas: $25 \mathrm{~km}, 5$ $\mathrm{km}$ e $1,66 \mathrm{~km}$.

Foram realizados um total de 24 experimentos considerando o período de 20 a 30 de maio de 2017. Em aspectos gerais, as simulações capturaram bem a quantidade e a distribuição espacial das chuvas extremas. Os esquemas de microfísica WSM3, WSM6 e Morrison-2mom apresentaram similares resultados de distribuição espacial da precipitação. O esquema YSU (PBL) foi o que apresentou os melhores resultados de distribuição temporal e espacial da precipitação, para qualquer parametrização de microfísica. Independente da configuração dos esquemas, a grade de resolução de 1,66 km foi a que apresentou os dados mais consistentes de simulação de quantidade de precipitação.

\section{Metodologia}

A pesquisa será de natureza aplicada e os experimentos serão realizados sobre um caso típico de evento extremo ocorrido no dia 14 de agosto de 2020 no oeste catarinense. O avanço de um sistema de linha de instabilidade desde o norte do Rio Grande do Sul e se deslocando pelo oeste catarinense levou à formação de diversos fenômenos intensos, como precipitação, ventos e granizo. A proposta é de estudo desse caso, no qual serão realizados experimentos com o modelo WRF com o objetivo de identificar o conjunto de parametrizações que melhor respondem à previsão dos eventos extremos associados.

A metodologia básica é executar o modelo WRF para diferentes parametrizações e os resultados serem submetidos a duas validações: a primeira é verificar os dados de saída perante dados observacionais obtidos de centros de previsão e de pesquisa; a, segunda; identificar qual parametrização impactará nos índices de estabilidade para formação de trovoadas intensas. Quando for possível, verificar com índices calculados a partir de dados observacionais.

O modelo WRF é constituído por diversos módulos e todos são de código fonte aberto e livre. O código pode ser compilado para sistemas Linux executando em sistemas computacionais de uso pessoal, como computadores e notebooks. O único impacto será no tempo de simulação, que é dependente da arquitetura dos processadores usados.

\section{Conclusão}

O trabalho de pesquisa ainda se encontra na fase de definição dos experimentos e na abordagem para quantificar os resultados. As dificuldades que poderiam advir estão relacionadas ao acesso a dados observacionais. Não tanto por óbices de empresas e institutos, mas, mais por falta de instrumentos de observação na área estudada. 


\section{References}

Bauer, P., Thorpe, A., \& Brunet, G. (2015). The quiet revolution of numerical weather prediction. Nature, 525(7567), 47-55.

Chawla, I., Osuri, K. K., Mujumdar, P. P., \& Niyogi, D. (2018). Assessment of the Weather Research and Forecasting (WRF) model for simulation of extreme rainfall events in the upper Ganga Basin. Hydrology and Earth System Sciences, 22(2), 1095-1117.

Comin, A. N., Justino, F., Pezzi, L., Gurjão, C. D. D. S., Shumacher, V., Fernández, A., \& Sutil, U. A. (2020). Extreme rainfall event in the Northeast coast of Brazil: a numerical sensitivity study. Meteorology and Atmospheric Physics, 1-22.

Efstathiou, G. A., Zoumakis, N. M., Melas, D., Lolis, C. J., \& Kassomenos, P. (2013). Sensitivity of WRF to boundary layer parameterizations in simulating a heavy rainfall event using different microphysical schemes. Effect on large-scale processes. Atmospheric research, 132, 125-143.

Moya-Álvarez, A. S., Estevan, R., Kumar, S., Rojas, J. L. F., Ticse, J. J., Martínez-Castro, D., \& Silva, Y. (2020). Influence of PBL parameterization schemes in WRF_ARW model on short-range precipitation's forecasts in the complex orography of Peruvian Central Andes. Atmospheric Research, 233-233.

Reboita, M. S. (2012). Retrieved from http:// repositorio.furg.br/handle/1/ 4742. Acessoem

Schwitalla, T., Branch, O., \& Wulfmeyer, V. (2020). Sensitivity study of the planetary boundary layer and microphysical schemes to the initialization of convection over the Arabian Peninsula. Quarterly Journal of the Royal Meteorological Society, 146(727), 846-869.

Shin, H. H., \& Hong, S. Y. (2011). Intercomparison of planetary boundary-layer parametrizations in the WRF model for a single day from CASES-99. BoundaryLayer Meteorology, 139(2), 261-281.

Skamarock, W. C. (2019). A description of the advanced research wrf version 4. ncar tech. Note.

Stensrud, D. J. (2007). Parameterization schemes: Keys to understanding numerical weather prediction models. New York, US: Cambridge University Press.

Warner, T. T. (2011). Numerical weather and climate prediction. Cambridge, UK: Cambridge University Press. 